\title{
The Case Study of Gestational Diabetes Mellitus (GDM) Underwent Elective Lower Segment Caesarean Section
}

\author{
Shaheena Salman A* \\ Assistant Manager OPD Services, Liaquat National Hospital and Medical College, Pakistan \\ Submission: May 6, 2017; Published: May 25, 2017 \\ *Corresponding author: Shaheena Salman A, Assistant Manager OPD Services, Liaquat National Hospital and Medical College, Pakistan, \\ Tel: +923333316730; Email: Shaheena.salman@lnh.edu.pk
}

\section{Introduction}

This case study is written for Family Health Practitioners and Nurse Case managers. To add, reflective critical analysis, frameworks, exploration of advanced role of case manager, ethical and professional issues which contribute enhancing the health and care needs, reflection on roles and responsibilities in relation to interdisciplinary teams is discussed in the report. It is to revitalize health systems in under-developed countries based on the tenets of the Alma Ata Declaration of Health Care, presented by the World Health Organization remains essential for attaining the maternal and child health related Millennium Development Goals (MGDs). Ehiri [1] informed that MGDs have a target date of 2015 and synthesize many of the commitments made in achieving that target. Reproductive health advocates have argued that MGDs require full implementation of the 'Program of action' for both the well-being of maternal and child through task forces. For this, Case Manager's role is pivotal where the right service for the right patient from admission to discharge is followed. Frater \& Leonard [2] deliberated case management stages and informed that the first step focuses on identifying clients and obtaining consent for managing the care and uplifting their existing health care needs. Also, consent was obtained for publication from the vulnerable high risk pregnant patient.

The extreme instabilities wide spreading the health care are inspiring a robust thoughtfulness in engaging patients at the native level of care to optimize cost savings and improvise outcomes. The natural collaborations occur when a local, multidisciplinary team support patients throughout the continuum of care in a transformative approach for delivering care [3]. Therefore, case management is widely explained in several numbers of steps to guide professionals to support families and clients at each level in holistic manner. Likewise other countries, Case Management Society of America established collaborative approach as the standard of practice and for attaining good patient care [4].
Further to elaborate, during the case study, components such as communication, conflict management, cooperation, coordination, independence, integrity, knowledge, mutual respect, optimism, shared responsibility and trust were explored. At initial step, informal consultation which focuses primarily on one health professional helping another address a specific clinical problem [5] was pondered. However, seeing the complexity of the case, intense collaboration was evident. This not only included a case manager to manage the case individually but involved professionals from across the continuum forming multi-disciplinary team to help patient's over lapping needs. ANA [6] emphasized that Nurses play an integral role in improving care outcomes through coordination. Therefore, role of a Nurse as being case manager is explored in the report. Frater \& Leonard [2] stated that the second step towards case management is the assessment and identification of opportunities which is explored throughout the case and intermittently. Arnold \& Boggs (2011) strikingly explained that 15 minutes interview, consisting of genogram; eco-map; therapeutic questions and commendations; provide a comprehensive look at family relationships. It helps in chasing family patterns. The structured format of these tools emphases on receiving relational data swiftly and can sensitize health practitioners to follow systemic family issues that affect patterns of health and illness.

Further, identification of multiple teams such as Obstetrician, Dietician, Endocrinologist, Physiotherapist and Home-health care nurse was done. Adding, co-provision model where accountability regarding patient's clinical situation is shared, supporting decision making from all team players and to overcome gaps in care was the motive of all individual stake holders in this case [4].

\section{Case Presentation}

A 38 years old lady with Gravida 5 Para 3+1, married since 11 years with previous 2 Caesarean sections, 1 forceps vaginal 


\section{Global Journal of Reproductive Medicine}

delivery and 1 miscarriage. During pregnancy, she was diagnosed as GDM. She was reluctant taking Glucophage tablet and noncompliant for sugar monitoring at home. She delivered healthy baby girl via Elective lower segment Caesarean section due to high sugar levels and chosen tubal ligation as a family planning method. To help patient maintain her regular sugar levels and medication regimen, mother-in-law was made realized to help patient taking care of her daily activities. This assessment was fully conducted in a form of family interview when patient visited hospital.

\section{Management and Outcome}

Frater \& Leonard [2] discussed that the third step of case management comprises of the development of case management plan in which the goals are established, interventions and prioritization of client's needs is determined for obtaining desired outcomes. Hence, looking at the below mentioned patient's course of complaint, evolution of goals, interventions and prioritization were drawn. To briefly explain, she was seen at 12 and 20 weeks' gestation and advised for oral glucose tolerance test which showed remarkably increasing trends.

\section{Discussion}

\section{Frameworks and concepts}

The case management is correlational to care delivery model. Lyon [3] put forward in literature that improved care coordination, deeper patient relationships and care plans that consider clinical, social, mental and financial factors are challenges that were over come through appropriate case-manager. These complexities primarily were sort by case-manager throughout the continuum of care and are salient features of the report.

Traditional models of case management within care are brokerage model, the managed care model and the integrated care model. In the case study, integrated model was utilized. This included integration of care management throughout the system of care, with provision of support and continuity of care which is evident from the literature [7]. It was possible for the multidisciplinary team to cater medical and social services such as procurement of self-sugar testing machine for patient. Together with counselling, monitoring of collaborative services, advocacy and continuous coordination were principally adhered. The merits of this model is that the case-manager had more authority than in a brokerage model but at times it was difficult denying services that were proposed by the team, this was the limitation though. Moreover, the risks for financial burden for family might have increased due to the involvement of multi-disciplinary teams. On the other hand, it is executed by Bernabei et al. [8] in randomized trial and proven that integrated social and medical care provide a cost effective approach by reducing re-admissions in hospital. Moreover, integrated services were the need assessment for the patient and so case-manager remained the first contact person to liaison with interdisciplinary teams. As evident from literature, integrated model targets patients who are at high risk of institutionalization, therefore case-manager tried to have less caseload and remained focused on this patient.

The focus was the on-going prevention of the progression of disease through interdisciplinary team. The already established network of providers was an ease for the client as well for the care outcome [7]. The client was followed through across the care settings for longer duration. On the other hand, if managed care model was approached, it usually resolves the current problem and is offered on a short-term basis. However, integrated care model is continued at a high level of intensity. Hence, as soon as patient was found to have GDM, case-manager managed her care till the case was disengaged. Comparing, in brokerage model case-manager spends less time directly with the patient to other two models. To highlight, assessments and re-assessments were done on continuous basis to scrutinize care plans, development of strategies to overcome concerns and to make a client a better fit-in at community level in integrated care model. The communication pattern was found to be stronger in between case-manager and service provider due to the similar organizational auspice.

\section{Case-manager's role as an Advanced Family Health Practitioner}

It is to articulate, Miodonski \& Hines [9] 'external focus advanced role' which appreciates when success of case management is seen through the customer's eyes. In such, casemanager with external focus will not only be patient advocates but will work collaboratively with physicians and team. In addition, external focus requires an awareness of the rapidly changing health care environment and the impact on case management.

The decisive ability, clear communication, consistent priorities, focused mind for anticipating barriers and proactively managing alternate solutions are a way forward for better management in future cases. Clear thinking is advised in a research by Miodonski \& Hines [9]. In relation to this, a critique to highlight here is the delayed decision making of a case-manager to involve mother-in-law for better social support of the patient. At the initial stage, emphasis on family dynamics and support was least prioritized that can be a learning point. To support, clear thinkers communicate succinctly and interact with patients and the health care team respectfully, professionally, and at the appropriate level. Adding, the optimism and enthusiasm to help client, generated new and creative ideas such as providing written instruction paper both for home (discharge summary/ report) and during hospitalization to refer her course of treatment and for better care outcomes such as instruction paper for medication timings, post natal care, dressing at operative site as a form of continuous reinforcement. It was feared that patient might end up into uncontrollable sugar levels in post-delivery state and likewise, any case-finding method needs to be able to identify individuals at high risk of future emergency admissions, coordinated care helped patient in maintaining her sugar levels 


\section{Global Journal of Reproductive Medicine}

[10]. Yet leaving no stone unturned in seeking out the solutions, addition of dietician, endocrinologist, and diabetic therapeutic nurse was managed as a part of intense collaboration [4,7].

\section{Predictive model of assessment}

Ross, et al. [10] explored number of tools and techniques that are inconsistent to this case report. The most accurate are predictive model that uses statistical algorithms to predict an individual's level of future risk of admission. Looking at the sugar levels which were a pre-dominant predictor for admission and even re-admission after patient was delivered was the core assessment that was performed. On the contrary, through strenuous team efforts patient was saved from post-delivery hospitalization. In practice, advance health practitioner uses a combination of a predictive model and clinical judgement. It is supportive to emblem individuals who are at high risk, and the case-manager then makes a judgement as to whether a person is likely to benefit from or not.

\section{Implementation and coordination of care activities}

Frater \& Leonard [2] after learning three steps of case management, fourth step is to keep the case management plan into action via coordinating care activities and implementing. Therefore, frequent visit of dietician and diabetes specialist nurse was coordinated. Birmingham \& Anctil [4] shared collaborative networking model to facilitate patient for home care and therefore, home-health nurse was introduced to visit the home for dressing, antibiotics, sugar status monitoring and pain management.

The competencies describe the advanced health nurse practitioner's ability to impart knowledge and associated psychomotor skills to patients and to whom the tasks are delegated. It involves the skills of interpreting and individualizing therapies through the activities of advocacy, modelling, and tutoring [11].

Also, physiotherapist's involvement for planning and implementing treatment course was appreciated as it helped preventing complications associated with GDM and Caesarean section. Moreover, incorporated family strengths as appreciated by Arnold \& Boggs (2012), viewing the family having strengths to cope with a problem, giving the family hope that a problem is not the end point rather only a circumstance in need of a solution and the more family is helpful, it is to get better outcome both mentally and financially.

Major responsibility of a case-manager is to deal managerial tasks regarding patient's pathway during the care continuum, coordination with multidisciplinary teams and being clinical expert in implementing plan of interventions was appreciative of the case [5].

Coordination of care requires inclusiveness, respecting other's views, excitement, loyalty and commitment. Similarly while reviewing literature, inclusive case-managers recognize their limitations and seek input from others' expertise [9].
That's the reason collaborative approach was made possible for deriving desired outcome. Further, it is explicitly shared in literature that those case-managers who have in-depth knowledge and credibility based on experience; appreciates learning and develops self are true aspirations as leader and being life-long learners.

It is necessary to attain qualification and training to develop an integrated plan incorporating all strategies, tools, resources, currently available and the tools and strategies that need to be developed. A well-versed individual can provide insight and guidance in conducting assessment and analysis, assist in steering internal politics, and act as a spokesperson and advocate for solving patients' cases [9]. The strong will to solve the case and to help individual and families is the driving force for case-managers. Therefore, when multi-disciplinary team was planning an early discharge without being focused on sugar levels, advocacy and striving force let the team knew that the patient requires attention and discharge activity needs to be well-planned. This step, not only helped patient revive timely but gave credibility to the case-manager for being real advocate. Acting as a patient's advocate involves numerous activities ranging from exploring self-awareness to engaging in social or health policy, steering or mediating communication this means keeping patient's needs and rights centrally to promote her self-determination and autonomy and to achieve the most appropriate services and outcomes.

Jimmy \& Jose [12] escalated that the therapies that are designed for individual patient needs to be implied stringently. Adherence to therapies is a primary determinant of treatment success. Otherwise, failure to it not only affects the patient but also the health care system. It may lead to substantial worsening of disease, death and increased health care costs. Therefore, integrated care model was utilized. A variety of factors are likely to affect adherence. It is necessary to improve medication adherence by scrutinizing specific barriers and adopting suitable techniques to overcome. Family support and little motivation persuaded patient to take medication and check blood sugar levels at home. It was explored and revealed that medication non-adherence occurred because patient perceived it to be unnecessary and due to fears and beliefs related to adverse effects of drugs. Hence, providing clear information is essential to improve adherence. So, a way forward for case-manager to incorporate this in future. Dobbels et al. [13] accentuated that medication adherence is the degree to which the person's behaviour corresponds with the agreed recommendations from a health care provider. To add on, though the terms adherence and compliance are synonymously used; adherence differs from compliance. Compliance is the extent to which a patient's behaviour matches the prescriber's advice. In contrast, adherence signifies that the patient and physician collaborate to improve the patient's health by integrating the physician's medical opinion and the patient's lifestyle, values and preferences for care. 


\section{Global Journal of Reproductive Medicine}

Jimmy \& Jose [12] proposed that medication counselling can be enhanced by providing detailed written information about medications. When patient was admitted, it was realized that brief written instructions which included drug cards, charts, written material sheet helped in improving adherence and acceptance in taking medications timely. The role of practitioner is to provide guidance and counselling regarding management of the condition timely [11].

It is pointed out by Jimmy \& Jose [12] that the potential burden of medication non adherence outcomes on health care delivery makes it an important public health concern. Barriers to the effective use of medicines specifically include poor providerpatient communication, inadequate knowledge about a drug and its use, not being convinced of the need for treatment, fear of adverse effects of the drug, long term drug regimens, cost and access barriers. In this case, it was assessed that the barriers were inadequate knowledge, motivation and fears.

Moreover, forgetfulness was the cause for not monitoring glucose levels at home, hence family role was introduced and behavioural support [13] was provided. The extent of non-adherence varies widely, and in different studies it has been recorded as low as $10 \%$ and as high as $92 \%$.Extensive review of the literature revealed that in developed countries adherence to therapies averages 50\% [13]. Likewise, Adisa \& Fakeye [14] experimented cross-sectional study using pretested questionnaire among 176 consented patients recruited from the endocrinology clinics of two teaching hospitals; and a retrospective review of case notes of the cohort for details of prescribed medications and blood glucose values. They reported non-adherence to prescribed anti-diabetes regimens has been identified as the most challenging aspect of diabetes control which continues to be a problem for healthcare providers, and a major concern for public health.

Overall, an appreciative point was that case-manager tried to make phone call to patient if she had checked her glucose levels. Since, at times accessibility was the issue for case-manager to patient's home therefore this strategy was found to be useful. The discussion on the topic with family and patient such as sharing of this information about the Hyperglycemia and Adverse Pregnancy Outcomes (HAPO) study which revealed that even mild hyperglycemia in pregnancy is associated with outcomes such as birth weight greater than the 90th percentile, increased rate of caesarean section and high cord plasma C-peptide levels which reflect foetal hyperinsulinemia [15] made her realize the importance of medication and checking of glucose regularly. Also, lifestyle modification and dietary changes with the assistance of a dietician were integral part of the case management. Moreover, this is to highlight that the Canadian Diabetes Association (CDA) guidelines for glucose tolerance test was utilized. As evident in literature $[11,15]$, case-manager formulates an action plan based on scientific rationale, evidence-based standards of care, and practice guidelines.

\section{Evaluation of the case management plan and follow- up}

Fifth step of case management by Frater \& Leonard [2] is the evaluation of plan and follow-up. The role of an advanced practitioner is to oversee the evaluation and satisfaction of client needs as well as the achievement of anticipated clinical outcomes from pre-hospitalization to post-hospitalization. The case-manager oversees that the patient receives appropriate care and services as outlined in the clinical pathway, plan within the allotted time frame [11].

\section{Reflective practice}

Evaluation can be reflected and as the concept of reflection is learnt, the practise comprises of the process where the individual thinks through a series of actions to identify positive and negative elements contributing to any situation in which they have been involved/ observed. Reflective practice is a critical component of good case management practice and can occur in a range of situations including problem solving and analysis. It is an integral part of good supervision in which joint exploration of some of the issues arising in practice should be encouraged in an environment of shared learning. It is in conversation with others that ideas are challenged, new approaches and perspectives can be considered and notions of what is possible and what is 'good practice' are developed and shared. The process involves problem solving from a point of having knowledge and understanding about something and applying analysis to this knowledge to inform future thinking and actions. It is an ongoing process in which an individual case-manager can draw on both the current situation and previous experiences to explore possible future action and consider the relative merits of any particular approach [16].

Management of case is not only to cure disease but to look into the aspects such as reduced cost, improved financial performance; reduced admissions and reduced length of hospital stay [17]. Hence, the contribution of multi-disciplinary team in managing care is worth noticing. If dietician does not provide appropriate teaching and dietary regimen to follow, think how the recovery is fasten? Same for diabetic care nurse who not only helped tracking of sugars in consultation with case-manager, obstetrician and endocrinologists but also motivated patient for taking meds on time and involved physiotherapist in care to take care of ambulation, exercises and daily activities.

\section{Ethical and inter professional issues}

Robertson [17] inscribed that there are numerous numbers of models of integration. Some are based on multidisciplinary teams. Others involve structural reform to create single, integrated organisations. The factors helping or hindering integrated working of the multi-disciplinary team fall into three categories: 1. Organisational issues: vision and culture, unified budgets or structures, communication and IT. 2. Professional issues: status and stereotypes, patient focus, training and education. 3. Policy 


\section{Global Journal of Reproductive Medicine}

and legal issues: policy environment, legislation, employment contracts, terms and conditions. NONPF [11] highlights that for decision making, information technology is pivotal. In contrast, it was observed that information technology had shut down in the setup where care-provision was in progress. Hence, referral which was informed via email or through computer based systems, case-manager made it possible to have that via manual written referral and information cards. Thus, care was not halt due to system breakdown but was incredibly managed through innovative approach as a part of patient-centred care [6] and problem-solving skills [18].

Lyon [3] interrogated that how do we build connectivity between case- managers, patients and the local care team? And in response to that it was suggestive to place nurse casemanagers within physical proximity of local providers to bridge the gap that occurs with the traditional model. This helps in identifying potential and existing issues. Fortunately, in this case, nurse case-manager remained in constant contact with patient and multi-disciplinary team.

This is to contemplate that it's a right of an individual to refuse for any treatment as per human rights-based model. On the contrary, not taking of the medication is injuries to foetus. Conflict occurs when the provider's duty is not in the best interest of the client. Therefore; utilitarian/goal-based model of ethical theory proposes to force mandatory institutionalization when a patient refuses to take medicine to protect other. Thus, goodness of an action over harm was the basic concept in this case (Arnold and Boggs, 2011). The client has a right to refuse, but its case-manager's role is to convince.

Theories incorporate concepts about autonomy and empowering clients to be responsible for self-care. However, what happens if the client's right to autonomy puts others at risk, such as baby? Whose rights take precedence? Literature suggests that laws may vary from state to state, but a moral principle should be universally applied. The moral principle of autonomy is to be explored in context when patient was offered the choice to decide for her family planning and tubal ligation was the mutual outcome from family and physician side. Some health issues such as tubal ligation have an associated stigma in Islamic culture [19]. On the other hand, Arnold and Boggs (2011) explained that autonomy is the clients right to self-determination. In the medical context, this is considered as fundamental ethical principle. It is the basis for the concept of informed consent, which means that patient and care provider makes rational and informed decision without coercion. Therefore, through effective communication skills as supported by NONPF [11], sensitive topic was addressed. Likewise, NONPF [11] elaborated case-manager's role to support a woman's right to make her own decisions regarding her health and reproductive choices within the context of her belief system.
ANA [6] intensify promoting the patient's autonomy. The case-manager facilitates decision making by mediating between the family through informing and supporting, considering needs and values. A hopeful development for future bilateral tubal ligation is supported by Macklin [20]. Thus, when it was advised in clinic to the patient to have tubal ligation, reluctance was expressed by her husband but after intellectual dialogues and discourse, husband learnt and permitted. It can be argued that in long term the decision for tubal ligation might have family controversies but it is suggestive to decide for family planning in this case seeing maternal grounds. Though it was difficult convincing patient's husband, still it was managed [20]. What are we to make of these assorted claims? Is the question of what ethical standard should prevail in international research? Is it an ethical domain like some others, in which people can disagree? Or are some of the opponents in this debate simply unreasonable, one good starting point would be to identify and seek to clear up ambiguities that lurk in the terms of these debate [20].

During the course of treatment, reflective analysis was made and concept of justice was applied deeply. Asking questions from self if it's fair or impartial to provide care appropriately? The related concept of equality such as just distribution of resources was a constant thought as in complex needs; human and material resources were utilized. Moreover, the instinct of providing best coordinated care mingled several team members which added up to have greater financial burden too. Hence, weighing the required care verses financial burden was discussed in depth with patient and family. With appropriate explanation of pros and cons and focusing patient as care-partner helped in resolving the case. Moreover, through utilizing skilled critical thinking concept and by using inquiry methods, supported well by Arnold and Boggs (2011) helped seeing the bigger picture. This inquiry method required problem solution in a systematic, organized and goal-directed way in making clinical decision.

\section{Multi-disciplinary team approach}

Multi-disciplinary team has received much attention now. Team members organise tasks so that associates from health and social care can work jointly with each other and with patients. Each member of the group is responsive of the patient's needs and what role the other partners play in delivering the most appropriate service [21]. Ham [22] apprises that integrated care have a single manager and use a single assessment process. Teams meet frequently, often daily, and focus on identifying the needs of the most vulnerable people managing their care in partnership with the individual's Physician. Each team has a health and social care co-ordinator that deals with referrals and acts as the single point of contact for the team. Co-ordinators provide the link between service users and team members, arranging care and support as necessary. It is intended that effective advanced health practitioner possess good relationships between patient and care team [17]. Along with this, much of the commentary 


\section{Global Journal of Reproductive Medicine}

on integrated care highlights the importance of a legislative and policy framework that consistently supports and encourages integration.

\section{Termination of the case management process}

Lastly, closure to the care, episode of illness termed as termination of the case management process. The focus of this step is to discontinue case management once the best possible outcome has been attained, the client transitions to the optimum level of function or the needs/desires of the client change [2]. It is imperative to evaluate client's status and goals with appropriate follow-ups via associated outcomes as the last step of case management [2]. It is to emphasize preparing client for discharge several visits before termination of case [19].

\section{Conclusion}

Miodonski \& Hines [9] discussed that by creating a 'case management leadership development program' for an organization can be an alternative to the process of external recruitment for case management department leaders. Such a program can be undertaken even in today's budget conscious environment by accessing existing resources in an organization in a creative manner. The authors outline an approach for management leaders to accept responsibility for succession planning and for case-managers to accept responsibility for promoting their own career development. The lack of wellqualified case management leaders will continue to be an issue for organizations for years to come as there is increasing pressure to reduce cost per case as a means to manage the budget. To turn this trend around, organizations must (1) recognize that there is a shortage of qualified and effective case management leadership across the country and (2) realize that there is a sufficient return on an investment in leadership development for case management, and the time for that investment is now. Much is already known about the factors determining effective integrated care and collaborative working but there remains a gulf between theoretical understanding and practical delivery [23]. Correspondingly, the motive for providing meaningful life should be an aim. The self-made model (Figure 1) shows the coordinated approach towards case management and by looking at the model, it is evident that the care that is provided to one patient is definitely complex and requires skill full personnel. The care model is core element of this case. It would not be possible without team efforts and collaboration and as rightly presented by Phaneuf [5] that the case-manager helps to attain better outcomes. However, a key barrier was time. Successful integration takes time and a short-term approach to policy making is likely to lessen the chances of successful integration [17]. Further, the qualities as mentioned by Phaneuf [24] for advanced health practitioner are sound clinical training and background, communication, integration (Figure 2), negotiation and problem-solving skills, ability to educate patients and other care providers, dexterity, in-depth knowledge of ethics and strength of character required to voice dissidence when necessary. These were taken care when case was managed.

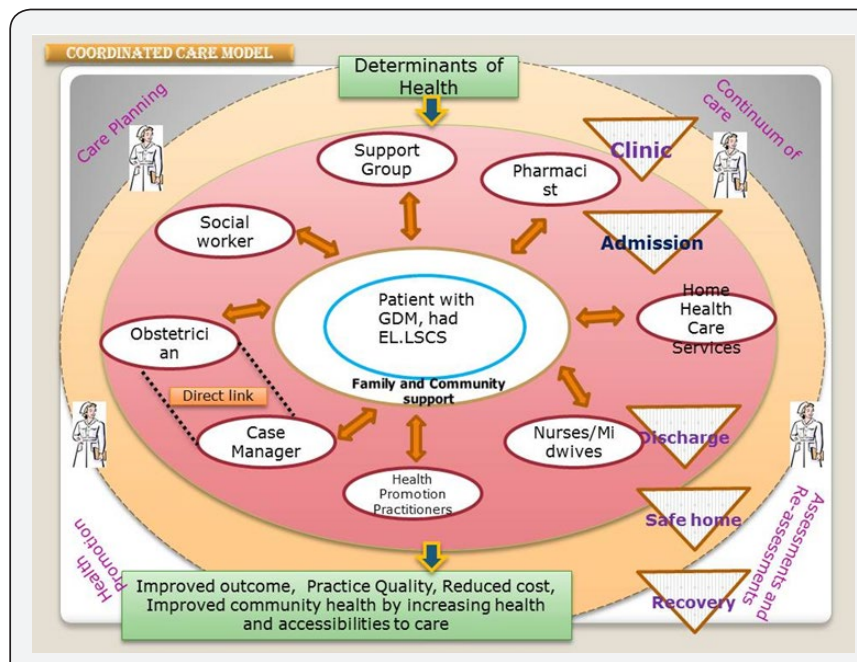

Figure 1: Model by Shaheena Salman Alwani.

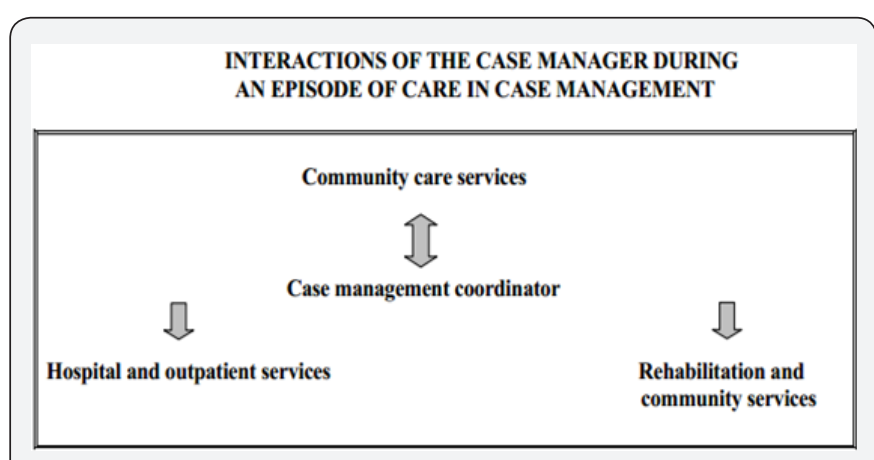

Figure 2: Interactions of the case manager during an episode of care in case management [24].

\section{Acknowledgement}

It is to thank my mentors and to certify that all material in this case study which is not my own is duly acknowledged.

\section{References}

1. Ehiri J (2009) Global challenges, programs and policies. In: John (Ed.), Maternal and Child Health, Springer, pp. 12-13.

2. Frater J, Leonard M (2010) Standards of Practice for Case Management. Case Management Society of America, pp. 1-30.

3. Lyon HD (2013) Transforming traditional case management through local provider partnerships. Optum, pp. 1-7.

4. Birmingham J, Anctil B (2002) Managing the dynamics of collaboration. The Case Manager 13(3): 73-77.

5. Phaneuf M (2008) The Roles and Qualities Required of a Case-Manager. Case Management- part 3.

6. ANA (2012) The Value of Nursing Care Coordination. A White Paper of the American Nurses Association, pp. 1-24. 


\section{Global Journal of Reproductive Medicine}

7. Scharlach A, Giunta N, Mills-Dick K (2001) Case management in long-term care integration. An Overview of Current Programs and Evaluations, pp. 1-94.

8. Bernabei R, Landi F, Gambassi G, Sgadari A, Zuccala G, et al. (1998) Randomised trial of impact of model of integrated care and case management for older people living in the community. BMJ 316(7141): 1348-1351.

9. Miodonski K, Hines P (2013) Leadership Development and Succession Planning in Case Management. Professional Case Management. Prof Case Manag 18(1): 5-12.

10. Ross S, Curry N, Goodwin N (2011) What it is and how it can best be implemented. Case management

11. NONPF (2002) Nurse Practitioner Primary Care Competencies in Specialty Areas: Adult, Family, Gerontological, Paediatric, and Women's Health. The National Organization of Nurse Practitioner Faculties.

12. Jimmy B, Jose J (2011) Patient Medication Adherence: Measures in Daily Practice. Oman Med J 26(3): 155-159.

13. Dobbels F, Van DR, Vanhaecke J, De-Geest S (2005) Growing pains: Non-adherence with the immunosuppressive regimen in adolescent transplant recipients. Paediatric Transplant. 9(3): 381-390.

14. Adisa R, Fakeye T (2013) Effect of number and type of antidiabetes medications on adherence and glycemia of ambulatory type 2 diabetes patients in south-western Nigeria. Pharm Pract (Granada) 11(3): 156 165.
15. Pallan S, Prebtani A (2011) Gestational Diabetes. 19(4).

16. Murray L (2010) Phases of case management: Community access ageing, disability and home care. In: Murray L (Ed.), Case Management Practice Guide 7. Department of Human Services NSW, p. 1-28.

17. Robertson H (2011) Integration of Health and Social Care: A review of literature and models, implications for Scotland. Royal College of Nursing, p. 1-42.

18. White P, Hall M (2006) Mapping the literature of case management nursing. J Med Libr Assoc 94 (2 Suppl): E99-106.

19. Riley JB (2012) Respect. In: Riley JB (Ed.), Communication in Nursing ( $7^{\text {th }}$ edn), Elsevier Publication, US, p. 97-98.

20. Macklin R (20012) Ethics in global health. In: Macklin R (Ed.), Research Policy and Practice. Oxford University press, pp. 160-198.

21. Niskanen JJ (2002) Finish care integrated? Int J Integr Care 2: e16.

22. Ham C (2010) Working Together for Health: Achievements and Challenges in the Kaiser NHS Beacon Sites Programme. Health Services Management Centre, University of Birmingham, England, p. 12.

23. Coxon K (2005) Common experiences of staff working in integrated health and social care organisations: a European perspective. Journal of Integrated Care 13(2): 13-21.

24. Phaneuf M (2008) The Roles and Qualities Required of a Case-Manager [Graphic] Interactions of the case manager during an episode of care in case management, p. 3 . 